\title{
The Sixth Series in the Spectrum of Atomic Hydrogen ${ }^{12}$
}

\author{
Curtis J. Humphreys
}

\begin{abstract}
The first member of the sixth series $\left(6 h^{2} \mathrm{H}^{\circ}-7 i^{2} \mathrm{I}\right)$ in the spectrum of atomic hydrogen has been observed at $12.37 \mu$, in agreement with the predicted position. Other newly observed lines include the second line of the Pfund series and the third, fourth, and fifth lines of the Brackett series. The source was a water-cooled discharge tube drawing 640 milliamperes at 5,000 volts. The tube was fitted with a $\mathrm{KBr}$ lens, which served also as a window for end-on observation. An atomic-hydrogen discharge was maintained by admitting water vapor through a Hopfield leak and pumping continuously. A conventional dispersing and recording system was used, consisting of a Perkin-Elmer model 12 spectrometer with a d-c breaker amplifier and Speedomax recorder. Relative-intensity measurements are included.
\end{abstract}

\section{Introduction}

The last new series to be discovered in the spectrum of atomic hydrogen was reported by Pfund ${ }^{3}$ in 1924 . This series originates in transitions to the fifth orbit from those of greater quantum number. This had been preceded 2 years earlier by the discovery by Brackett ${ }^{4}$ of the series originating in transitions to the fourth orbit. Both of these contributions followed closely and probably received considerable impetus from the spectacular experiments of Wood, ${ }^{5}$ in which he succeeded in exciting exceptionally long Balmer series in hydrogen discharges. This spectrum was of unusual purity, and, because of the striking contrast between the brilliant lines and dark background between, was called the "black discharge." Brackett inferred correctly that the excitation conditions favoring the "black discharge" were prerequisite to the production of series resulting from transitions to levels higher than the second or third. This conclusion depends on the fact that the length of series and the appearing of series having a high level as the final state are both dependent on the density of population of the more highly excited states.

The extension of the hydrogen spectrum farther into the infrared region to include additional series predicted by theory has long constituted a challenge. This has become increasingly insistent when one considers that in more than a quarter of a century of intensive infrared activity, marked by spectacular developments in observing techniques, there has been no further infrared extension of the hydrogen spectrum. A review of the present state of our knowledge of this spectrum will be followed by a discussion of the experimental conditions required for the excitation of higher series and utilized in the current experiments. Of the many compilations of hydrogen data, one of the most satisfactory is that given by Grotrian. ${ }^{6}$ The Paschen series was observed, in the range of photography, as far as the eighth member by Poetker. ${ }^{7}$ Brackett observed

\footnotetext{
Curtis J. Humphreys, J. Opt, Soc. Am.42, 432 (1952).

2 Presented at the Symposium on Molecular Structure and Spectroscopy, Ohio State Tniversity, 1952 .

3 A. H. Pfund, J. Opt. Soc. Am. 91, 193 (1924).

4 F. S. Brackett, Astrophys. J. 56, 154 (1922)

5 R. W. Wood, Proc. Roy. Soc. (London) [A] 97, 455 (1920); Phil. Mag. 42, 729 (1921).

${ }_{6}$ W. Grotrian, Handbuch der Astrophysik, Kap. 5, Gesetzmässigkeiten in der Serienspektren, p. 475 (Springer, Berlin, 1930).

7 A. H. Poetker, Phys, Rev. 30, 418 (1927).
}

only two members of his series, and Pfund found only the first member of the series bearing his name. Table 1 is introduced to point out the locations of the first two members of known and predicted series. The latter include all entries below Pfund- $\alpha$. Predicted positions of the higher members of infrared series, including and beyond that of Brackett, are given by Swensson. ${ }^{8}$

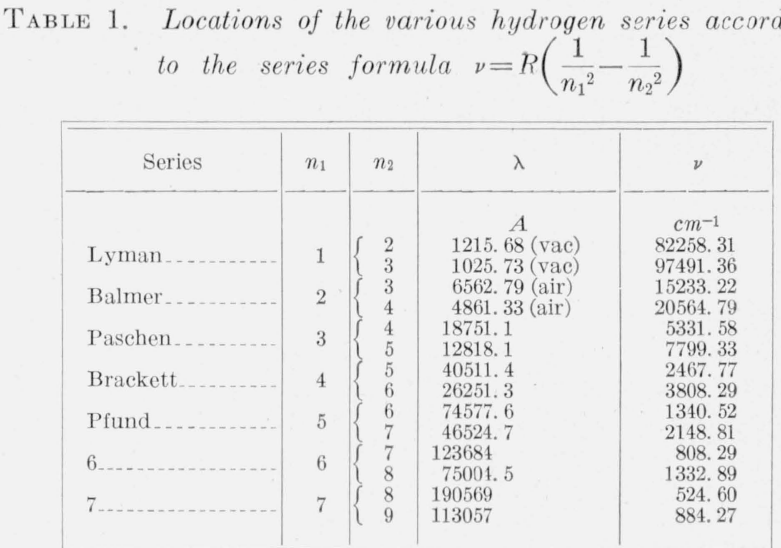

Results of a new investigation presented and discussed here include observation of the first member of an entirely new series, $6 h^{2} \mathrm{H}^{\circ}-7 i^{2} \mathrm{I}$, a second member of the Pfund series, and three additional members of the Brackett series beyond the two originally reported.

\section{Experiments}

The method of exciting the hydrogen spectrum adopted in these experiments was similar to that employed by earlier observers mentioned in the references, consisting of a high-current Geissler discharge. Modifications were introduced to meet the special requirements of the observations, the most important of which was the necessity for using a transparent window on the discharge tube to permit emergence of energy associted with the first line of the new series at $12.37 \mu$. All reported observations of the hydrogen spectrum emphasize the difficulty of maintaining an atomic spectrum because of the tendency of hydrogen atoms to associate

8 J. W. Swensson, Prédictions de raies atomiques dans l'infrarouge, extrait des Mémoires in $-8^{\circ}$ de la Société Royale des Sciences de Liége, quatrième série, XI, fase. I (Louvain, 1951). 


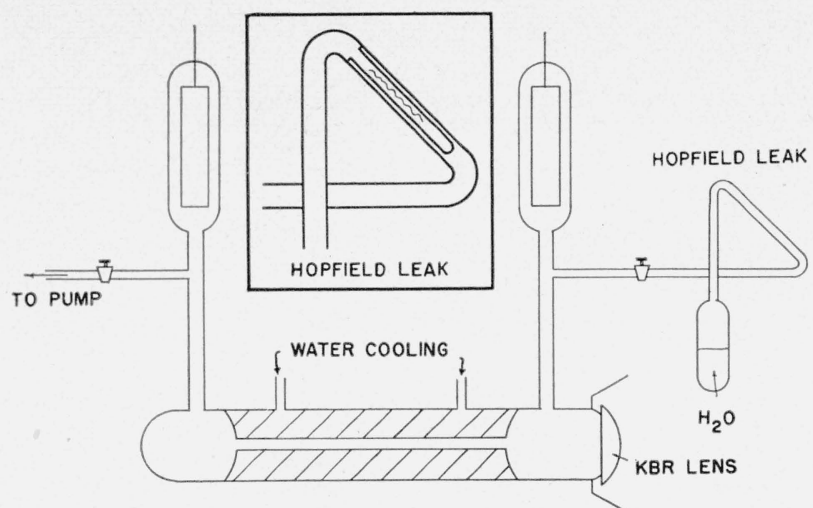

FIGURE 1. Schematic design of source tube and Hopfield leak.

to form $\mathrm{H}^{2}$ molecules, with the attendant development of the many-line molecular spectrum. This association is favored in the vicinity of the electrodes and is probably caused by a catalytic action by the metal. Wood's successful excitation of a pure atomic-hydrogen discharge apparently resulted from two features of his technique: (1) construction of an extremely long tube, of which only the central portion was used for end-on observation through a thinlyblown bend, and (2) provision for continuous change of the hydrogen supply, accomplished by connecting a constantly operating exhaust pump to the system, and at the same time admitting hydrogen through a capillary tube drawn to a fineness determined by experiment to give the optimum pressure.

More recent investigations have pointed to the advantage of using moist hydrogen. This is made obvious by the appearing of the intense Balmer series in a vacuum system when the last traces of moisture are being pumped out. This condition favoring the atomic spectrum is probably caused by liberation of hydrogen in the atomic state upon dissociation of the water molecule. Arnulf and Dunoyer $^{9}$ described the design of a tube for producing atomic-hydrogen discharges of high intensity, featuring a side tube containing gypsum and from which small amounts of moisture could be liberated into the system by heating.

For the current series of experiments, several tubes were built and tried out, all of which had the advantageous features established by earlier investigators to the extent permitted by the practical requirements of the work. The desirable characteristics included provision for cooling, associated with ability to withstand steady high currents, satisfactory attachment of a suitable transparent window, and geometric design to provide a region of maximum intensity and to permit illumination of large-aperture optics. The tube used in obtaining the reported data is illustrated schematically in figure 1. The principal differences between this and other preliminary designs is in the size of the central discharge tube. The improved intensity resulting from a relatively small capillary, approximately 4 $\mathrm{mm}$ in diameter, more than makes up for the small

A. Arnulf and L. Dunoyer, Rev, Optique 9, 510 (1930). size of the image projected onto the slit of the spectrometer. The really unique feature of the source operation was the manner of admitting hydrogen. Because the use of wet hydrogen has been demonstrated to be advantageous, the idea of introducing pure water vapor suggested itself. This proved to be successful and also made the replenishment of hydrogen extremely simple. As indicated, a small tube of distilled water was attached to the source through a Hopfield leak. This leak, illustrated in figure 1, consists of a crack generated in the small blind-end tube sealed inside the figure-4 shaped tube with a ring seal. The opening of the crack is controlled by a clamp at the crossover point of the tubing. In practice, the rate of vapor flow was increased somewhat by mounting a radiant heater near the water supply. The lens of $\mathrm{KBr}$ on the end of the tube was used in the final version, replacing a flat window. This is one of the lenses supplied for a Perkin-Elmer model 21 spectrometer, which had not been installed owing to prevailing low humidity. Actually, the lens made possible a considerable increase in the amount of energy that could be admitted to the optical system. In addition to the water cooling the tube was wrapped in cotton, over which a continuous stream of water flowed. The best development of higher series was found to be realized when the tube was operated at the lowest pressure for which a steady discharge could be maintained.

All other components in the setup were conventional commercially available pieces of apparatus. The source of power was a $2 \frac{1}{2}-\mathrm{kva}$ transformer. The output voltage was rated at 5,000. The transformer was slightly overloaded at the maximum source current of $630 \mathrm{ma}$. A variable-voltage autotransformer of 40-amp capacity was used to control the power supply.

A Perkin-Elmer model 12 spectrometer with a rock-salt prism was used as the dispersing system, modified only by removal of the housing over the source optics to permit advantageous placement of the source, and attachment of a simple motor-driven gear train for scanning. In the later phases of the work, an improvised housing was mounted so as to enclose the end of the light source and projecting system to permit filling the entire optical path with oil-pumped nitrogen for the purpose of eliminating water vapor. For the preliminary experiments a Hornig thermopile, as used in the early Perkin-Elmer models, was used. This was later replaced by one of the fast-response a-c couples in current production. A d-c system was used throughout the experiments for amplifying and recording the voltage output. This consisted of a Liston-Folb breaker amplifier and a Speedomax recorder. All of the visible components are readily identifiable in figure 2 .

It is apparent that the experimental setup does not differ markedly from that used by previous observers, particularly in regard to operating characteristics of the source and specifications of the dispersing system. The use of an amplifying and recording system does, however, provide a margin of sensitivity that could not be matched in the older 


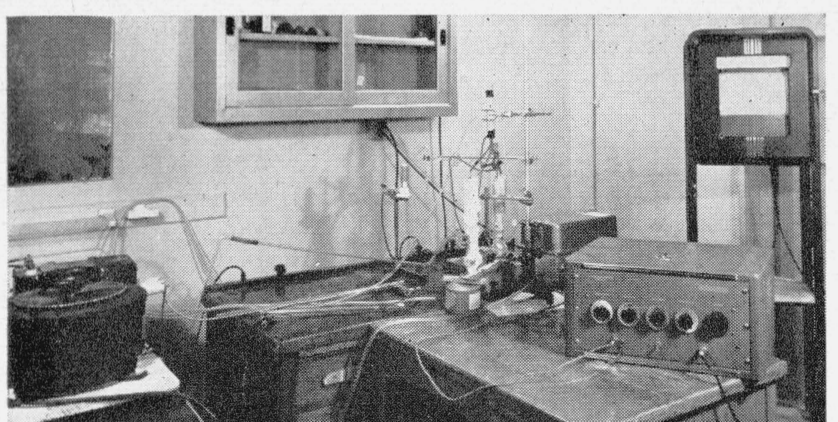

FIGURE 2. Layout of apparatus.

point-by-point observations with a galvanometer.

The observation of the first line of the sixth series was accomplished after considerable preliminary experimentation to achieve optimum conditions, including optical adjustment to obtain maximum signal strength, choice of the most suitable component ratings in the improvised filtering circuit placed between the amplifier and recorder; and selection of the most satisfactory amplifier gain. The replacement of the window with a lens and the substitution of the a-c thermopile were made after observation of the new line had been confirmed by several records. These changes resulted in a marked improvement in signal strength, making it possible to reduce the slit width from $1.7 \mathrm{~mm}$, necessary to obtain the first indications of the line, to $0.5 \mathrm{~mm}$, the settings used to obtain the records reproduced in figures 3 and 4 .

Most of the records were run with a gain such that a $0.1-\mu \mathrm{v}$ signal gave a deflection of from 30 to 40 percent of full scale on the chart. The profiles shown in reproduction of actual records appear rather broad. This is mainly because available speed-changing gears did not permit selection of optimum ratio of scanning speed to record-chart speed. Of course, the slit widths that necessarily had to be used were much too great to permit development of true profiles. The wavelength calibration of the spectrometer was accomplished by using well-known emission and absorption sources, including a mercury lamp, atmospheric carbon dioxide and water vapor, and a polystyrene film. The determination of the wavelength of the new line is not to be regarded as precise, because fairly broad absorption bands were used for calibration in the $12-\mu$ region. It is precise enough for certain identification, but the numerical wavelength listed is the calculated value. As an extra precaution, to make sure that the new line was genuine, records were run with a $\mathrm{CaF}_{2}$ filter in front of the slit to eliminate all radiant energy of wavelength greater than 12 including the new line, and with a scattering filter to remove all energy of wavelengths shorter than about $6.5 \mu$ and to permit the new line to pass.

\section{Discussion}

Figures 3 and 4 are reproductions of records of the first line of the new series, selected from a large number of similar records. The scale of ordinates shown on each figure is to show the relation of de-
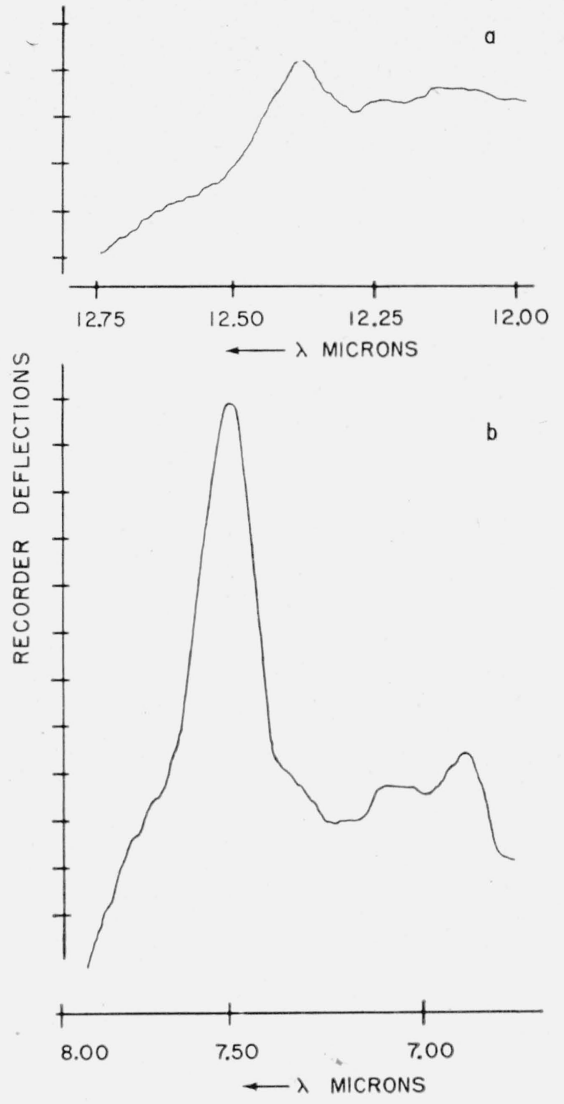

Figure 3. First, or $\alpha$, lines of $(a)$ sixth series and $(b)$ Pfund series, in the infrared under identical conditions (stit $0.5 \mathrm{~mm}$., signal $0.1-\mu v$ full scale on recorder chart).

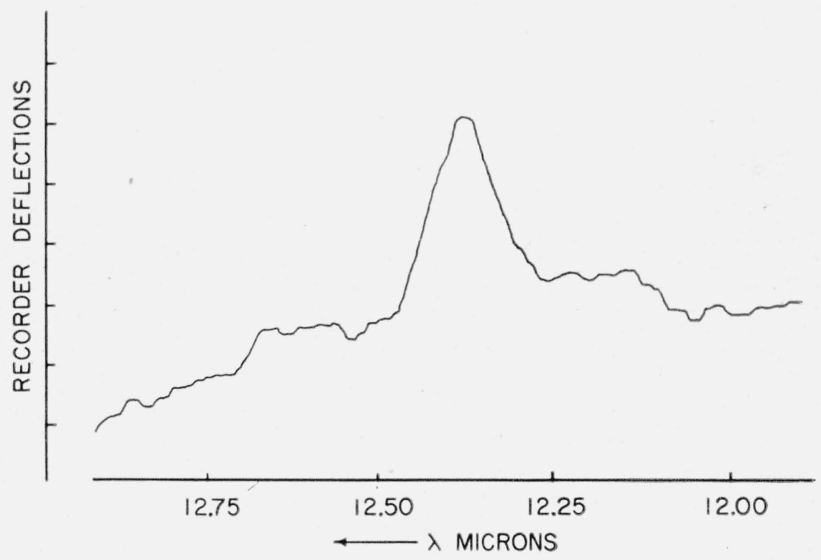

FIGURE 4. First, or $\alpha$, line of sixth series, in infrared, observed under optimum conditions (slit $0.5 \mathrm{~mm}$ ).

flection to chart width, one division being equivalent to five scale divisions on the chart. Figure 3 represents a considerable improvement over the record súbmitted with a recent letter, ${ }^{10}$ but was obtained by using a similar program of observation, namely, to record the new line and the Pfund line under the same conditions in order to compare intensities. Figure 4 shows the best record obtained to date.

\footnotetext{
10 J. Opt. Soc, Am, 42, 432 (1952).
} 

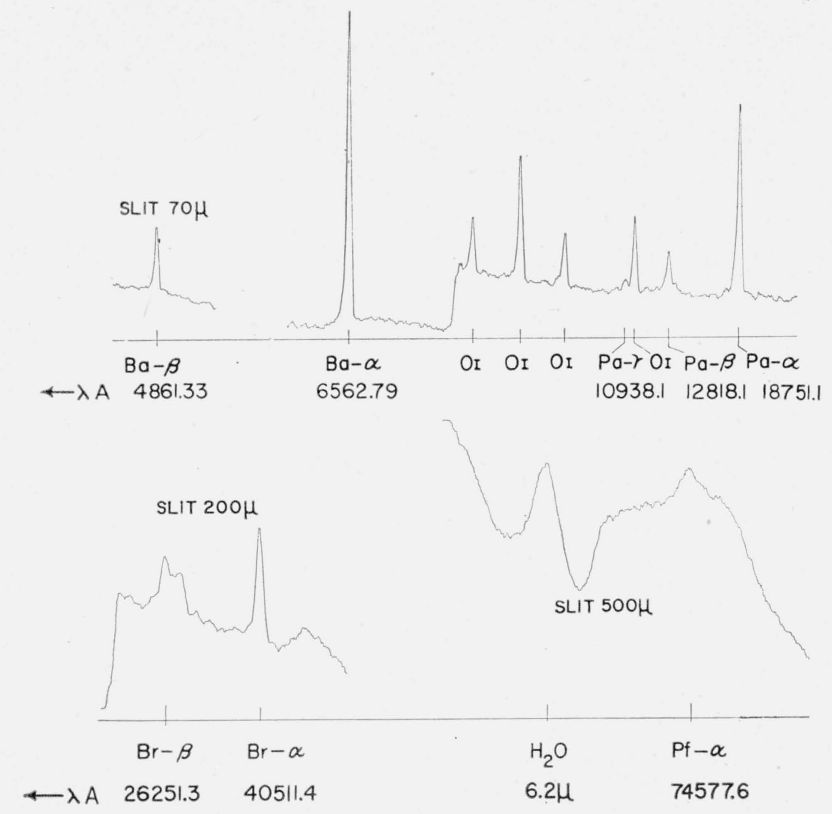

Figure 5. Record covering interval from Balmer- $\beta$ to Pfund- $\alpha$.

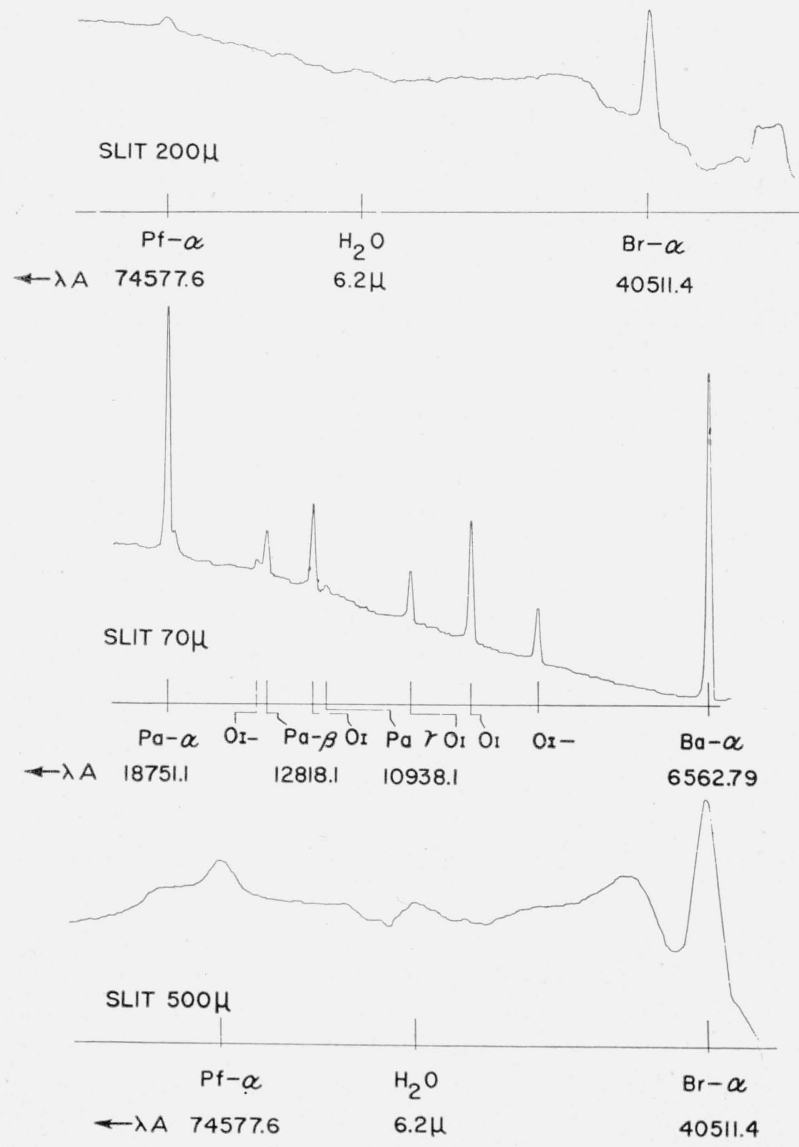

Figure 6. Records covering interval from Balmer- $\beta$ to Pfund- $\alpha$, showing effect of removal of water vapor from optical path.
Although the observation of the sixth series of hydrogen was the principal experimental objective, additional records were obtained for the purpose of extending known series and obtaining intensity comparisons within and between series. The entire spectral range available with the rock-salt prism installation was covered. This range extended from below Balmer- $\beta$ to somewhat beyond the new line of the sixth series. Survey records described in the appropriate captions are reproduced in figures 5 and 6.

Figure 5 shows the entire region from Balmer- $\beta$ to Pfund- $\alpha$, with some featureless sections removed. At the top is shown the first two members of the Balmer series, illustrating the intensity decrement. This is followed, after adjustment for drift, by a record of the region from Balmer- $\alpha$ to Paschen- $\alpha$. Three members of the latter series may be picked out, with a fourth probably distinguishable from noise. The other profiles are unresolved multiplets of $\mathrm{O}_{\mathrm{I}}$. The lower section covers the region including Brackett- $\alpha$ and Pfund $-\alpha$. The slit width was increased from 70 to $200 \mu$ after traversing Paschen- $\alpha$, and the recording was again interrupted to widen the slit to $500-\mu$ and adjust the zero after passing Brackett- $\alpha$. The recording was made without any attempt to remove $\mathrm{H}_{2} \mathrm{O}$ vapor from the path, and the curve shows the characteristic profile of the $6.2-\mu$ water-vapor band with a slit too wide to reveal rotational structure.

Figure 6 , representing a record run in the opposite direction, covers the same features. There is slightly less noise because of the fact that the a-c thermopile of greater sensitivity was used, permitting operation on lower gain settings for the same slit openings. This run was made with dry nitrogen in the spectrometer and in the improvised housing enclosing the source optics. The $6.2-\mu$ water-vapor band can hardly be seen. The upper and middle sections are part of a continuous run. The lower section illustrates the condition of partial removal of water vapor.

Table 1 shows that Pfund- $\beta$ is located at wavelength $4.65 \mu$. On the basis of decrements found in other series, it is expected to be about one-sixth to one-seventh as intense as Pfund- $\alpha$ and about 2 percent of Brackett- $\alpha$. Observation of Pfund- $\beta$ under conditions of the earlier experiments (Brackett, Pfund) was quite hopeless because of inadequate sensitivity, coupled with the fact that it would have been completely masked in the wide-slit image of Brackett- $\alpha$ at $4.0 \mu$. Figure 7 shows the results of a very limited amount of work with a $\mathrm{PbTe}$ cell. The Pfund- $\beta$ line can clearly be seen to the left of Brackett- $\alpha$ with intensity close to that estimated. In the lower curve the same line is shown with increased amplifier gain. In this case overloading prevented a satisfactory representation of the profile of Brackett- $\alpha$. A difficulty of observing with a telluride cell in this region is that the maximum of temperature radiation from the tube comes at about $5 \mu$. The telluride detector is very sensitive to this continuous background. The trouble was overcome by greatly diminishing the power input to the source at some sacrifice of signal strength. 


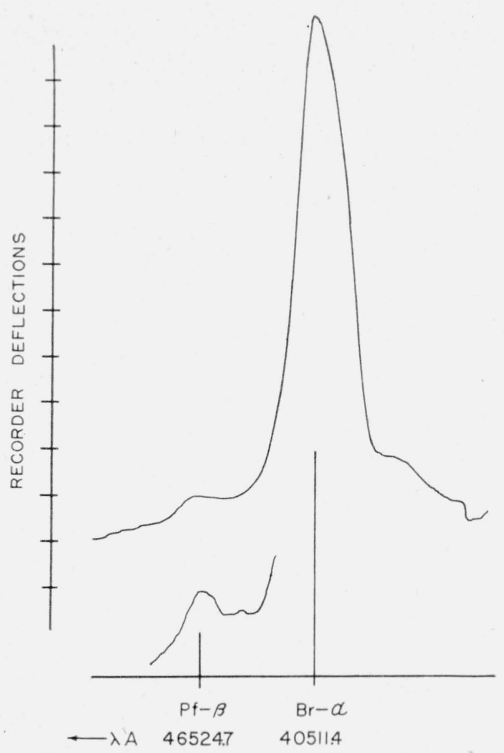

Figure 7. Records showing Pfund- $\beta$ and Brackett- $\alpha$, as observed with a $\mathrm{PbTe}$ detector (slit $400 \mu$ ).

The fact that all of the Brackett series beyond the first member lies in the wavelength region of maximum sensitivity of lead-sulfide photoconductive cells made the exploration of this series beyond the second member, as observed by Brackett, appear promising. Figure 8 shows the development of this series as observed with a $\mathrm{PbS}$ detector. Brackett- $\alpha$ is not included, being out of the range of sensitivity. The $\beta$ - and $\gamma$-lines are well developed. Paschen- $\alpha$, falling just between Brackett- $\delta$ and Brackett- $\epsilon$, partly overlaps these lines with the scale of dispersion inherent in the system. It is certain that they could be clearly separated with a high-resolution spectrometer. As in the other records shown, the lines not identifiable with the hydrogen spectrum originate in $\mathrm{O}$, as would be expected when water vapor is dissociated in the source. In the lower record Paschen- $\alpha$ and its companion on the short wave side are attenuated by using a different gain setting. The upper short section displaced vertically only is a subsequent run without attenuation, starting just beyond but not including Paschen- $\alpha$.

A final record, figure 9 , is inserted to show the development of the Paschen series under the experimental conditions described and with a $\mathrm{PbS}$ detector. The series may be traced to six members. Poetker photographed it up to and including the eighth member. It appears certain that better results could be obtained with a grating instrument because of the possibility of separating weak hydrogen and oxygen lines by utilizing both higher dispersion and greater resolving power. The stronger lines, including Paschen- $\alpha$ were attenuated. T'The gain setting was changed in some instances after the profiles had started to develop, or before the background level was reached. This is the explanation of the sharp pips adjacent to some profiles.

The work on hydrogen to date is to be regarded as preliminary to more detailed investigation, which will include precise evaluation of intensities, search

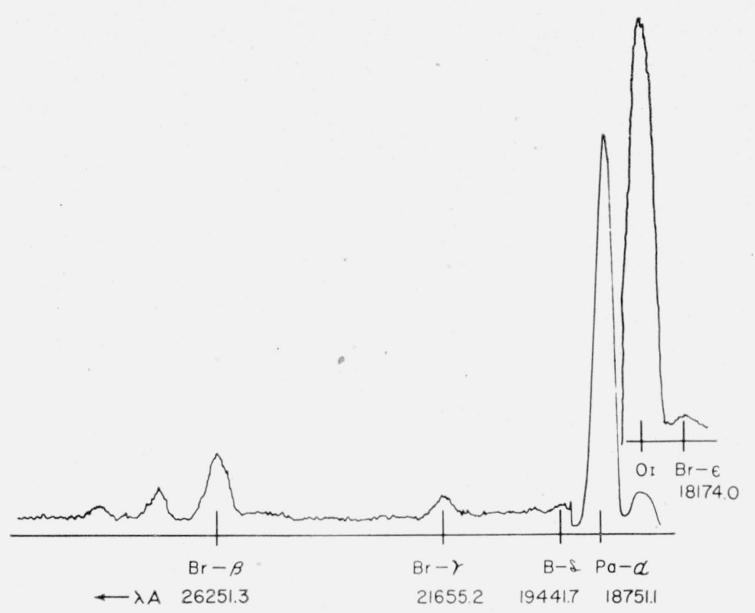

Figure 8. Records showing the higher members of the Brackett series as observed with a $\mathrm{PbS}$ cell (slit $50 \mu)$.

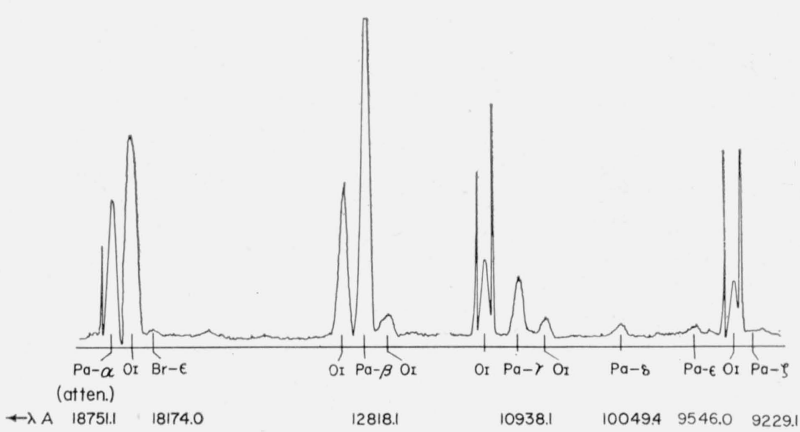

Figure 9. Record of Paschen series observed with a PbS cell $($ slit $50 \mu)$.

for new series, extension of known series, and determination of excitation conditions required for maximum development of infrared series. The study of the excitation of the hydrogen emission spectrum is of great astrophysical interest in its relation to the theoretical explanation of the long Balmer series with small intensity decrement that occur in the solar chromosphere, stars with extensive gaseous envelopes, and planetary nebulas. A brief discussion of this matter with pertinent reference material is given by Kiess and Humphreys ${ }^{11}$ in a report on the intensities in the flash spectrum observed at the solar eclipse of 1940. Correlation of astrophysical with laboratory data is needed. Brackett i2 regarded the hydrogen spectrum produced in his laboratory source as arising to a large extent from inelastic collision without ionization. He offered an explanation that was supported by the unexpectedly high ratio of the intensity of Paschen- $\alpha$ to Balmer- $\alpha$, this reaching 4 to 3 in Brackett's experiments. This ratio was not attained in these experiments, the maximum Paschen- $\alpha$ to Balmer- $\alpha$ being 4 to 5 , but even this is surprising, considering the behaviour of the other series.

The most significant information for the inter-

${ }^{11} \mathrm{C} . \mathrm{C}$. Kiess and C. J. Humphreys, Nat. Geographic Soc., Contributed

Tech. Papers, Solar Eclipse Ser. 2, p. 53 (1942).
12 F. S. Brackett, Astrophys. J. 56, $154(1922)$ 
pretation of excitation conditions responsible for any observed hydrogen spectrum is to be found in intensity relationships, including decrement ratios within a series and intercomparisons between different series. Radiometric measurements with a thermopile readily lend themselves to intensity determinations owing to the essentially nonselective character of this detector. Table 2 gives a summary of the relative intensities of several hydrogen lines as estimated from the observations. Except for instances where photoconductive cell observations were made within relatively narrow wavelength intervals, as, for instance, the region from Brackett- $\beta$ to Brackett- $\epsilon$, these intensity comparisons are based on thermopile observations and should be reasonably precise, subject to some corrections, including those for reflection and scattering by the optical parts. These corrections, which vary with wavelengths have not been made in the preparation of this report. The exit slit was imaged within the boundaries of the receiver for all slit widths used.

Over a year ago E. U. Condon suggested to the author that it would be of great interest to intercompare intensities of hydrogen lines originating in a common upper state. Such intercomparison of course provides data for evaluating relative probabilities of transition to various lower states. The data of table 2 may be used to provide such information.
ТАВLE 2. Observed relative intensities of hydrogen lines

\begin{tabular}{|c|c|c|}
\hline Line & $\lambda$ & $\begin{array}{l}\text { Relative } \\
\text { intensity }\end{array}$ \\
\hline $\begin{array}{l}\text { Balmer- } \boldsymbol{\beta} \\
\text { Balmer- } a\end{array}$ & $\begin{array}{c}\text { A } \\
4861.33 \\
6562.79\end{array}$ & $\begin{array}{r}150 \\
1000\end{array}$ \\
\hline $\begin{array}{l}\text { Paschen } \delta \\
\text { Paschen } \gamma \\
\text { Paschen- } \beta \\
\text { Paschen- } \alpha\end{array}$ & $\begin{array}{l}10049.4 \\
10938.1 \\
12818.1 \\
18751.1\end{array}$ & $\begin{array}{r}6 \\
28 \\
140 \\
700\end{array}$ \\
\hline $\begin{array}{l}\text { Brackett- } \gamma \\
\text { Brackett- } \beta \\
\text { Brackett- } \alpha\end{array}$ & $\begin{array}{l}21655.2 \\
26251.3 \\
40511.4\end{array}$ & $\begin{array}{r}13 \\
40 \\
120\end{array}$ \\
\hline $\begin{array}{l}\text { Pfund- } \boldsymbol{\beta} \\
\text { Pfund } \boldsymbol{\alpha}\end{array}$ & $\begin{array}{l}46524.7 \\
74577.6\end{array}$ & $\begin{array}{r}3 \\
20\end{array}$ \\
\hline Sixth series- $a \ldots$ & 123684 & 3 \\
\hline
\end{tabular}

For instance, the first line of the sixth series, Pfund- $\beta$, Brackett- $\gamma$, and Paschen- $\delta$, all originate in a common upper level. The table shows that these intensities fall within the same order of magnitude. Mechanical limitations of the spectrometer prevented extending this array to Balmer- $\epsilon$. This intercomparison is pointed out as a very sketchy beginning of something that should be carried out more precisely and in greater detail.

In conclusion, the author acknowledges with thanks the valuable help of L. G. Mundie and A. J. Cussen in all phases of the experimental work.

Corona, Calif., September 3, 1952. 